\title{
Philonsorbonne
}

14 | 2020

Année 2019-2020

\section{Husserl et la conception de la " nature »}

\section{Mariana BARDELLI}

\section{(2) OpenEdition}

\section{Journals}

Édition électronique

URL : https://journals.openedition.org/philonsorbonne/1344

DOI : 10.4000/philonsorbonne.1344

ISSN : 2270-7336

\section{Éditeur}

Publications de la Sorbonne

\section{Édition imprimée}

Date de publication : 14 février 2020

Pagination : 11-23

ISSN : 1255-183X

\section{Référence électronique}

Mariana BARDELLI, « Husserl et la conception de la « nature » », Philonsorbonne [En ligne], 14 | 2020,

mis en ligne le 01 mars 2020, consulté le 08 juin 2021. URL : http://journals.openedition.org/

philonsorbonne/1344 ; DOI : https://doi.org/10.4000/philonsorbonne.1344 


\title{
Husserl et la conception de la « nature »
}

\author{
Mariana BARDELLI
}

Si la conception de la nature n'est pas un thème majeur dans l'héritage de la pensée husserlienne, elle ne doit cependant pas être négligée dans la mesure où à partir des années 1920, elle revient souvent comme thème dans les réflexions du philosophe. Le débat sur le concept de nature est d'abord le point de départ de la critique formulée par Husserl contre les sciences de la nature, et ensuite un enjeu très important dans le développement du concept de monde de la vie, notion majeure de la pensée du «dernier» Husserl. Notre propos est donc de clarifier l'évolution de l'approche husserlienne de la notion de nature qui a fait entre autres l'objet de ses cours du semestre d'été de 1919 et de 1927. Le but est d'abord de comprendre la critique husserlienne des «sciences objectives", notamment en ce qui concerne leur conception de la nature. Dans un deuxième temps, nous pouvons passer à la signification positive que Husserl lui donne. Toutefois, comme nous allons le voir, c'est un double sens de la nature qui apparaît dans les textes des années 1920 et 1930. Nous essayerons de montrer en quoi consiste cette ambiguïté. Ce propos est d'autant plus important qu'il nous permet de mieux comprendre l'ambiguité du concept de monde de la vie (Lebenswelt) et celle de l'esthétique transcendantale qui sont en lien strict avec ce double sens de nature, comme le souligne Julien Farges dans un article intitulé «Monde de la vie et primordialité chez Husserl. Les ambiguïtés de la conception phénoménologique de l'esthétique transcendantale »" 
Par ailleurs, cette ambiguïté à la fois du concept de monde de la vie et de la notion de nature rend possible une confusion entre ces deux notions dans la mesure où le monde de la vie peut désigner dans certains cas un monde primordial, et où la nature au sens large signifie le monde prédonné à l'intuition. Julien Farges clarifie cette confusion créée par l'identification entre monde de la vie et monde primordial chez Husserl. Il précise par ailleurs que l'ambigüité d'un sens prégnant et d'un sens élargi de la conception phénoménologique de l'esthétique transcendantale «s'enracine manifestement pour Husserl dans le double sens de la notion de nature $»^{2}$.

Ces considérations justifient davantage notre présente tâche, à savoir clarifier ce double sens de la notion de nature et distinguer davantage cette ambiguiité de celle du monde de la vie. Comme nous le verrons, cette corrélation confuse entre ces deux concepts est soutenue par le fait que la prédonation passive est comprise à la fois comme prédonation de ce qui est originaire et de ce qui est immédiatement là, de sorte qu'elle est fermement liée à la problématique de l'esthétique transcendantale. Nous exposerons ainsi le mouvement dans lequel Husserl aborde et développe trois sens $\mathrm{du}$ concept de nature: la nature objectivée des sciences de la nature qui la définissent comme un référent spatio-temporel contenant l'ensemble des choses du monde; par le biais de l'exercice de l'approche phénoménologique en revenant à la couche fondatrice de toutes les autres, nous avons affaire à une nature originaire, mais aussi à une nature prédonnée dans la passivité du sujet comme nature vécue. Dans un troisième temps, nous exposerons une nouvelle conception de la nature formulée par Husserl dans certains manuscrits tardifs, et révélée par la traduction française récente de l'un de ces manuscrits. Ce nouveau concept de nature, comme nous le verrons, est une alternative au double sens selon lequel il s'agit soit de la nature qui fonde l'expérience soit de la nature vécue dans l'expérience du monde. Notre approche a cette particularité de focaliser sur l'ambiguïté de la notion de la nature qui est une clé pour mieux comprendre l'ambiguïté du monde de la vie et celle de l'esthétique transcendantale.

Dans le second tome des Recherches phénoménologiques pour la constitution $^{3}$, la question de la nature s'insère dans le cadre d'une analyse statique sur les différentes couches de la constitution, dont font partie la nature matérielle et la nature animale, ainsi que le monde de l'esprit. Toutes ces couches ont, dans la nature physique, leur fondement en tant que couches des « simples choses ». Dans certains textes plus tardifs tels que La Crise des sciences européennes et la phénoménologie transcendantale ${ }^{4}$, Husserl prolonge la critique de la notion de "nature » telle qu'elle est conçue par les sciences de la nature. Il en formule une tout autre conception qui figure notamment dans des textes comme les Leçons du semestre d'été de 1919 (intitulées Natur und Geist) et Expérience et jugement. Nous verrons que

2. Ibid., p. 21.

3. Désormais abregé Ideen II.

4. Désormais abrégé Krisis. 
ce parcours constitue une remise en question d'une certaine approche de la «nature », et aboutit à une pensée de la nature dans le cadre d'une phénoménologie génétique ainsi que d'une science générale de l'esprit. La nature est ainsi niée en tant qu'instance objective qui possède une existence en soi détachée du monde subjectif. D'autre part, elle est affirmée comme couche qui fonde toutes les autres, mais aussi comme « monde naturel» tel qu'il nous est immédiatement donné comme notre réalité.

Husserl commence le tout premier paragraphe des Ideen II en donnant la définition suivante : la nature est «l' "univers" spatio-temporel en totalité, le domaine tout entier de l'expérience possible »; et un peu plus loin : la nature « est le champ des réalités transcendantes, c'est-à-dire spatio-temporelles ${ }^{5}$. Il revient ainsi à la définition de la nature qui sert à l'intérêt des chercheurs des sciences objectives dans l'attitude qui leur est propre, à savoir l'«attitude naturaliste». Celle-ci s'oppose à l'«attitude axiologique» et à l'« attitude pratique » en tant qu' «attitude théorique », par laquelle « une "aperception" souveraine détermine à l'avance ce qui est nature au sens de la science de la nature et ce qui ne l'est pas $»^{6}$. Ce qui a été nommé jusqu'à présent «nature » correspond à «l'expérience accomplie dans cette attitude " et, en ce sens, la nature "désigne une sphère de "pures et simples choses" $\gg{ }^{7}$. Husserl affirme que cette définition ne prend pas en compte d'autres objets d'une connaissance possible, en mettant à leur place une abstraction qui sépare cette sphère de pures et simples choses des valeurs, des évaluations, de tout le sens donné aux objets par l'attitude pratique, de sorte que « [1]a nature en tant que simple nature ne contient pas de valeurs, ni d'œuvres d'art, etc. $[\ldots] »^{8}$.

Est ainsi posée la possibilité d'accéder, dans le cadre d'un intérêt purement théorique, à la notion de nature en tant que monde de pures et simples choses. Or pour ce faire, il est nécessaire de procéder à une abstraction des prédicats des sphères axiologiques et pratiques. Pourtant, il sera affirmé plus tard dans la Krisis que les objectités spatio-temporelles sont chargées de valeurs, de caractères pratiques, avant tout intérêt théorique. Ce qui est conçu par le sujet théorique comme «monde objectif», comme « monde vrai » se révèle donc être « une substruction théorético-logique » ${ }^{9}$

5. E. Husserl, Recherches phénoménologiques pour la constitution, trad. fr. Éliane Escoubas, Paris, Presses Universitaires de France, 2004. p. 23 [1]. Dans les notes du présent article, la pagination indiquée en premier lieu est celle de la traduction française. La pagination entre crochets droits renvoie à l'édition des Husserliana: Hua IV : Edmund Husserl gesammelte Werke. Ideen zu einer reinen Phänomenologie und phänomenologischen Philosophie. 2, Phänomenologische Untersuchungen zur Konstitution, Haag, Martinus Nijhoff, 1952.

6. Ibid., p. 24 [2].

7. Ibid., p. 50-51 [25].

8. Ibid., p. 25 [3].

9. E. Husserl, La Crise des sciences européennes et la phénoménologie transcendantale, trad. fr. Gérard Granel, Paris, Éditions Gallimard, 1976, p. 44 [130]. La pagination entre crochets droits renvoie à l'édition des Husserliana: Hua VI. Edmund Husserl gesammelte Werke. Die Krisis der Europäischen Wissenschaften und die Transzendentale Phänomenologie, Haag, Martinus Nijhoff, 1962. 
(Substruktion), c'est-à-dire une construction qui est naturalisée de telle sorte qu'elle nous apparaît comme si elle était pré-donnée dans l'expérience de façon originaire. Par l'attitude théorique, nous mettons à la place du monde des "vérités constructibles ", qui pourtant ne peuvent pas être éprouvées, qui ne sont pas perceptibles, donnés à l'intuition. En procédant ainsi, nous faisons seulement l'expérience de la «couche de matérialité spatiotemporelle », et réduisons l'expérience vis-à-vis des hommes et des sociétés à sa seule matérialité.

La nature n'est pas le «monde vrai», comme peuvent le croire les chercheurs en science objective, qui n'adhèrent à cette conception que par pure idéalisation. Cependant, en affirmant cette thèse, Husserl ne refuse pas complètement le concept de nature au sens scientifique, il l'accepte mais en lui redonnant sa place dans l'ordre des couches de la constitution à partir d'une ontologie matérielle. Dans ce cadre, la nature physique, c'est-à-dire la nature objet des sciences objectives, reste affirmée comme champ de la localisation spatio-temporelle des choses et des corps, de sorte qu'elle joue le rôle de champ de possibilités réales pour la vie psychique du sujet. Dans l'analyse de la sensibilité pour laquelle le corps propre (Leib) est l'instance matricielle, la nature physique constitue le soubassement en tant que champ où se localisent le corps propre et la vie psychique dans l'espace et le temps objectifs. Selon Husserl, le corps propre a une réalité double, il est subjectif autant que physique, et l'âme en tant que liée à un corps a également une place dans la réalité physique. Husserl conçoit ainsi le corps propre et l'âme comme parties de la nature physique, de sorte que même le "je pense " est «déterminé par le contexte substantiel-causal de la nature, qui n'est précisément pas la simple nature physique, alors même que la nature physique est celle qui fonde et qui co-détermine toute autre nature $»^{10}$. La nature physique « est là pour nous comme fondement des corps propres, des sensibilités et des vies psychiques ${ }^{11}$. Mais si le corps propre et la vie psychique du sujet sont, d'un côté, localisés physiquement, spatiotemporellement, alors la nature n'a pas d'existence en soi comme étant vraie - comme le posent les sciences objectives -, mais seulement comme couche fondatrice qui présuppose donc les autres.

Dans les Ideen II, ce qui est compris comme nature, au sens étroit, coïncide avec la nature physique comme couche placée au niveau inférieur de la constitution en tant que «monde matériel donné à l'intuition». Au sens large, la nature est «le monde tout entier de l'attitude naturaliste $»^{12}$, mais cela ne peut être défini que d'un point de vue théorique et par la procédure d'une abstraction. Ainsi, Husserl rectifie le sens que les sciences objectives lui donnent, en montrant que ce sens étendu de nature est en fait le corrélat des actes de l'attitude naturelle, à partir de laquelle l'attitude théorique opère

10. E. Husserl, Recherches phénoménologiques pour la constitution, op. cit., p. 256 [181].

11. Ibid., p. 258 [182].

12. Id. L'attitude naturaliste, qui n'est pas à confondre avec l'attitude naturelle, est celle des chercheurs en sciences de la nature. 
une abstraction. La nature "se présente ici en tant que pur sens des actes qui constituent l'attitude naturelle, en tant que son corrélat constitutif $\rangle^{13}$. En ce sens, la nature ainsi objectivée est constituée par des actes des sujets égologiques, et en effet elle ne peut qu'être posée par des actes intropathiques. Husserl affirme alors la relativité des choses par rapport aux sujets dans la mesure où la nature intersubjectivement commune « est une unité d'apparitions qui est posée et doit être posée par des sujets, à savoir qui doit être posée dans des actes rationnels $»{ }^{14}$. Ainsi, la nature n'est pas un «étant en soi », mais elle «a besoin d'un complément», elle est toujours relative.

Dans la Krisis, la notion de Lebenswelt étant un objet central des analyses husserliennes, la question de la nature vis-à-vis des sciences objectives reparaît dans le cadre de la discussion sur la possibilité d'une approche scientifique du monde de la vie. En celui-ci, écrit Husserl, nous sommes des objets, et cela "avant toute constatation scientifique », alors même que "nous sommes pour ce monde des sujets [...] qui ont de ce monde une expérience ${ }^{15}$, qui donnent au monde son sens à travers ses expériences, ses évaluations, etc. Dans l'attitude théorique, nous sommes pour ce monde des objets comme les autres, en même temps que dans l'attitude pratique, nous lui donnons à chaque fois son sens en tant que sujets «qui s'en inquiètent, qui l'évaluent»: "Ce monde de la vie est le monde spatio-temporel des choses, telles que nous les éprouvons dans notre vie pré- et extra-scientifique, et, au-delà de cette expérience, telles que nous savons qu'elles peuvent y être éprouvées. Nous avons un horizon de monde en tant qu'horizon d'une expérience possible de choses. Les choses ce sont : les pierres, les animaux, les plantes, aussi les hommes et les formations humaines; mais tout cela est là sur le mode subjectif-relatif $[\ldots] »^{16}$. Selon les descriptions exposées dans la Krisis, le monde de la vie qui contient les animaux et les hommes est à la fois la couche de la spatio-temporalité des choses et la couche pratique. La séparation entre les corps physiques et les produits des actes pratiques peut être effectuée par abstraction, mais dans notre expérience du monde de la vie, ils ne sont prédonnés qu'ensemble. C'est-à-dire que pour cette approche, le monde de la vie ne coïncide pas avec la couche sensible, bien qu'il l'inclue en lui, mais c'est un monde déjà pénétré par la signification culturelle. Husserl affirme que le monde donné d'avance est l'horizon qui inclut toutes nos fins, tous nos actes, toutes nos possibilités pratiques, tous nos thèmes théoriques. Ainsi, dans la Krisis, la signification culturelle du monde s'impose à l'expérience sensible que nous en faisons, pour autant qu'il n'y a pas de séparation entre l'une et l'autre: «C'est à lui - le monde de l'intuition

13. Ibid., p. 248 [174].

14. Ibid., p. 241 [171].

15. E. Husserl, La Crise des sciences européennes et la phénoménologie transcendantale, op. cit., p. 157 ; Hua VI, p. 141.

16. Id. 
qui "éprouve" effectivement - qu'appartient la forme spatio-temporelle avec toutes les figures corporelles qui s'inscrivent en elle, c'est en lui que nous-mêmes nous vivons, conformément à notre mode d'être, c'est-à-dire dans toute la chair de notre personne ${ }^{17}$.

Le développement de la notion de Lebenswelt dans la Krisis ne mène pas à une réduction à la couche de substrat sensibles. Dans cet ouvrage, le but de Husserl est de montrer le monde de la vie comme fondement des constructions logiques. Ce qui n'est pas le cas d'Expérience et jugement, dont l'introduction annonce la présente tâche, à savoir chercher "le plus élémentaire » dans l'expérience qui fonde l'acte de jugement : «l'expérience la plus simple est celle des substrats sensibles, de la couche naturelle du monde concret total $»^{18}$. En faisant l'abstraction de l'attitude personnelle ${ }^{19}$, Husserl arrive à la «pure nature universelle $»^{20}$. Celle-ci est comprise à présent comme l'étant dont on fait l'expérience simple et immédiate dans notre rapport au monde, qui en tant que tel est le monde «de tous les corps », des "substrats sensibles». Cet étant est donné "comme corps naturel », sans que l'intérêt ou l'acte du sujet soient en jeu. Ce qui revient à dire que ce substrat est prédonné dans la «pure passivité ». Cette nature, écrit Husserl, est «la couche inférieure, celle qui fonde toutes les autres ${ }^{21}$. Il reprend ainsi la définition des Ideen II selon laquelle la nature au sens prégnant est la strate qui fonde le monde réal.

La nature invoquée à présent en tant que couche fondatrice est à la fois la nature physique, et le champ de pré-donation du sujet. À la différence de la Krisis, Husserl distingue le monde dont nous faisons l'expérience, qui n'est pas la simple nature, et le substrat des perceptions prédonné au moi dans la passivité. Il distingue donc ce que nous percevons comme corps et ce que nous percevons comme nature psychique liée à un corps : "Tout ce qui est mondain et extérieur, nous le percevons comme un étant corporel, situé dans la totalité spatio-temporelle de la nature. Là où nous rencontrons des animaux et des hommes, des objets culturels [...], nous n'avons pas la simple nature, mais l'expression d'un sens spirituel $»^{22}$. À présent est donc affirmée cette séparation des deux sphères de perception, celle de la «pure

17. Ibid., § 9, p. 59 [50].

18. E. Husserl, Expérience et jugement, trad. fr. Denise Souche-Dagues, Paris, Presses Universitaires de France, 2001, p. 73.

19. Les choses que l'on ne trouve pas dans la nature physique et dont le sens est constitué par des actes d'évaluation et des actes pratiques sont prises en considération dans l'attitude « personnaliste » exposée par Husserl dans les Ideen II. À la différence de l'attitude théorique, dans l'attitude personnaliste, le moi est toujours intéressé par les objets autant qu'il est affecté par eux. En elle, nous sommes en rapport avec les choses, comme notre environnement et non en tant que nature objective. Cf. E. Husserl, Recherches phénoménologiques pour la constitution, op. cit., p. 258 ; Hua IV, p. 182-183.

20. E. Husserl, Expérience et jugement, op. cit., p. 66 [57]. La pagination entre crochets droits renvoie à l'édition allemande: E. Husserl, Erfahrung und Urteil: Untersuchungen zur Genealogie der Logik, Hrsg. Von L. Landgrebe, Claassen \& Goverts, Hamburg, 1948.

21. Ibid., p. 63 [54].

22. Ibid., p. 63-64 [55]. 
perception sensible » et celle qui inclut les prédicats pratiques et logiques. Selon ce point de vue, la nature est le champ prédonné pour toute activité du moi - ce qui inclut la "réceptivité » et l'affection. Ainsi, elle n'est pas subjective en tant qu'étant constituée par le sujet, mais en tant qu'étant pour le sujet. Si la « limitation abstractive » à la couche fondatrice du monde n'est valable que pour moi ${ }^{23}$, c'est parce que la nature sensible m'est prédonnée dans la passivité, sans qu'elle ait besoin d'être valable pour les autres sujets.

Dans l'introduction d'Expérience et jugement, le monde expérimenté dans l'attitude naturelle est réduit à l'étant «invariant de toute la relativité changeante des appréciations qui portent sur lui $»^{24}$. Husserl affirme que l'originarité du monde de la vie «ne peut être mise en évidence que par la destruction de ces couches de sens $»^{25}$. Le résultat de l'analyse par cette méthode de « déconstruction» $(A b b a u)$ est «la totalité spatio-temporelle de la nature ». En ce sens, Husserl affirme, dans le $\S 12$ que tout ce dont nous faisons l'expérience comme ayant quelque propriété que ce soit est «donné dans une expérience sensible immédiate ${ }^{26}$. Une considération comme celle-ci a ouvert la possibilité à l'interprétation - comme l'ont fait certains commentateurs ${ }^{27}$ - selon laquelle le monde de la vie coïncide avec la couche de pure perception. Cette confusion est aussi due au fait que si la nature peut être affirmée comme strate qui fonde toutes les autres dans la mesure où tout ce qui se trouve dans le monde a une matérialité spatiotemporelle, nous n'en faisons pas une expérience intuitive. Elle ne peut nous être révélée que par une réduction, qui mène à une abstraction. Bruce Bégout, en problématisant cette approche, observe que cet accès à «la

\section{Cf. Ibid., p. 66 [57]. \\ 24. Ibid., p. 63 [54]. \\ 25. Ibid., p. 60 [51]. \\ 26. Ibid., p. 62 [53].}

27. Les commentateurs qui admettent cette interprétation ont affirmé la signification culturelle du monde de la vie face à son substrat naturel comme l'héritage de la pensée du dernier Husserl. En ce sens, cf. P. Janssen, Geschite und Lebenswelt, ein Beitrag zur Diskussion von Husserls Spätwerk, Nijhoff, Den Haag, 1970; I. Kern, «Die Lebenswelt als Grundlagenproblem der objektiven Wisenschaften und als universales Wahrheits und Seinsproblem ", in R. Bernet, I. Kern, E. Marbach (Hrsg.), Edmund Husserl, Darstellung seines Denkens, Hambourg, F. Meiner, 1989, p. 199-208, (trad. fr. par Ph. Cabestan, in Alter. Revue de phénoménologie, 6, 1998, p.419-431); J. Benoist, «Le monde pour tous: universalité et Lebenswelt chez le dernier Husserl », in Recherches husserliennes, vol. 5, p. 27-52, 1996. B. Bégout écrit à ce sujet : "Il semble donc que l'ambiguité initiale qui existait dans la détermination du monde de la vie, tantôt compris comme a priori esthétique de l'expérience sensible [...] tantôt comme monde prédonné de l'expérience culturelle, celle de 1'“attitude personnaliste" des Ideen II, tend à disparaître au profit de la seconde qualification. Progressivement la signification culturelle de la Lebenswelt s'impose et nécessite la prise en considération de sa dimension sociale et historique » (B. Bégout, L'Enfance du monde, Les Éditions de la Transparence, 2007, p. 73). En contestant que ce rapprochement entre le monde de la vie et la nature a eu lieu chez Husserl, Julien Farges souligne qu'il y a plutôt une confusion dans la mesure où le "caractère d'intuitivité immédiate» est revendiqué par Husserl «à la fois pour le monde de la vie et pour l'expérience sensible». Cf. J. Farges, «Monde de la vie et primordialité chez Husserl. Les ambiguïtés de la conception phénoménologique de l'esthétique transcendantale », op. cit., p. 27. 
couche naturelle de l'expérience perceptive du monde de la vie » ne se fait que par «une méthode de construction de l'originaire $»^{28}$. Ainsi se pose-t-il la question de savoir si le monde expérimenté dans l'attitude naturelle, et réduit à sa pure donation sensible, n'est pas une nouvelle idéalisation.

D'une part, Husserl pointe bien que cette procédure n'est pas la même que celle des sciences objectives. Il refuse que la nature puisse avoir une existence indépendante du monde de la vie. Alors que les sciences de la nature prennent pour objectif quelque chose qui ne l'est pas et posent l'existence en soi de quelque chose qui n'existe pas séparément, la réduction phénoménologique à la couche de la pure perception prend en compte l'originarité de la totalité spatio-temporelle par rapport aux autres couches de la constitution sans faire de celle-ci une entité autonome. Cependant, cela n'est révélé que par une procédure qui est elle-même " anti-naturelle » dans la mesure où elle me donne quelque chose qui n'est pas opérant en tant que tel dans ma vie en dehors de la réduction.

La nature semble donc jusqu'ici évoquée dans ces deux sens différents chez Husserl. À l'idéalisation opérée par les sciences de la nature, il substitue un double sens qui sert à deux interprétations possibles du rapport du monde de la vie à la nature : le concept prégnant de nature comme réalité physique qui englobe tous les objets réaux, gagné par l'abstraction de toute signification; et un sens étendu de la nature comme « univers naturel » qui désigne la «totalité des réalités extensives coexistantes ${ }^{29}$, et en cela «nature et réalité se recouvrent» ${ }^{30}$. Cette ambiguité crée une perméabilité entre ces deux concepts, puisqu'il arrive au monde de la vie d'être identifié à un monde primordial et à la nature d'avoir son sens étendu au tout des réalités.

Nous devons à présent aborder la différence entre la prédonation du monde de la vie et la prédonation de la nature afin de mieux distinguer ces deux concepts et de mieux comprendre cette ambiguïté qui est finalement celles des modes de la prédonnée, et est donc une problématique strictement liée à l'esthétique transcendantale. Ici, il est important de revenir sur la différence entre l'originel: le pré-donné dans l'intuition en premier; et l'originaire : le fondement premier ${ }^{31}$. Selon l'interprétation du monde de la

28. B. Bégout, L'Enfance du monde, op. cit., p. 72.

29. E. Husserl, Natur und Geist : Vorlesungen Sommersemester 1919, Boston-London, Kluwer, 2002, p. 135.

30. Ibid., p. 136.

31. Cf. E. Husserl, «À propos du concept d'originalité », Hua XIV, 389; trad. : Sur l'intersubjectivité I, Paris, PUF, 2001, p. 110. Cf. aussi J. Farges, «Monde de la vie et primordialité chez Husserl. Les ambiguïtés de la conception phénoménologique de l'esthétique transcendantale»,op. cit. Julien Farges précise que, chez Husserl, l' " originalité » est « toujours rapportée à l'expérience perceptive de la donation d'un objet comme présent "en chair et en os" » (p. 16). "Le néologisme "primordinal" (primordinal), venant désigner le caractère originaire de l'originalité en question laisse entendre quelque chose comme une "ordinalité" qui renvoie manifestement à un ordre de fondation entre les différents degrés d'originalité » (p. 17). 
vie comme instance essentiellement culturelle exposée dans la Krisis, nous ne faisons pas l'expérience intuitive de la couche de substrats sensibles, mais du monde tel qu'il nous est immédiatement prédonné. La spatio-temporalité qui appartient au sens d'être du monde de la vie est celle «de la vie $»^{32}$. Dans la Krisis, il s'agira de montrer, par le biais de l'épochè transcendantale, que ce dont je fais l'expérience originellement est en fait le résultat d'une « formation de sens originaire ${ }^{33}$ active et par la suite sédimentée : le monde de la vie prédonné dans l'intuition n'a pas le caractère originaire qu'il semble avoir ${ }^{34}$ dans sa prédonation. La réduction transcendantale opérée dans la Krisis le reconduit aux «origines intentionnelles» qui sont au fondement de la formation de son sens. Dès lors qu'il n'est plus question de la «nature » subjectivement constituée, il sera montré que le monde de la vie est lui-même intersubjectivement constitué, valable pour tout un chacun. S'il est prédonné dans la passivité de l'expérience intuitive, il se révèle être intersubjectivement constitué à un niveau plus profond de la constitution, donc le produit d'une activité préalable. Il ne s'agit pas d'une prédonation originelle de l'originaire, mais de ce qui prédonne ce qui a été antérieurement constitué dans une expérience plus originaire. L'opération analytique effectuée à présent se distingue de celle de l'introduction d'Expérience et jugement, où la dimension originaire de la couche fondatrice du monde est révélée par abstraction. Cependant, dans l'attitude naturelle nous ne faisons pas une expérience immédiate, originelle, de l'originarité de cette nature fondatrice.

Il y a donc dans le cas de la nature comprise comme nature physique et le monde de la vie une non-coïncidence entre ce qui est premièrement éprouvé dans la présence immédiate et ce qui est constitué en premier. Nous comprenons ainsi pourquoi l'ambiguïté de ces deux notions peut ébranler cette distinction et créer une confusion entre ces deux concepts qui est en réalité la confusion entre originalité et originarité, entre pré-donation et primordialité. La nature fondatrice est moins originale que le monde de la vie, qui malgré sa moindre originarité à l'égard de la nature est pré-donné à l'intuition comme ce qui est le plus immédiat. Comme ce qui est éprouvé dans la présence immédiate, le monde de la vie se donne à la perception en tant que conscience "originale» d'un être présent. Selon Husserl, le «lui-même» des modes de l'évidence se trouve "présent dans ces intuitions comme ce qui peut être éprouvé et confirmé effectivement dans l'intersubjectivité, et aucune substruction n'est ici pensable ${ }^{35}$.

32. E. Husserl, La Crise des sciences européennes et la phénoménologie transcendantale, op. cit., p. 191 ; Hua VI, p. 171.

33. Cf. Ibid., § 40 ; B. Bégout, L'Enfance du monde, op. cit., chap. 3.4.

34. Nous pourrions encore noter que les sciences objectives n'opèrent pas seulement une abstraction - comme dans le cas d'une approche «naturalisante » du monde de la vie - mais elles opèrent une construction théorique de l'originaire, en produisant un originaire complètement idéalisé.

35. E. Husserl, La Crise des sciences européennes et la phénoménologie transcendantale, op. cit., p. 145 ; Hua VI, op. cit., p. 130. 
Si l'éprouvabilité et la confirmation effective appartiennent au mode d'évidence du monde de la vie mais pas à celui des constructions logicothéoriques, c'est que ces dernières se fondent sur la Lebenswelt. Pour cette raison, le monde de la vie peut être à la fois non originaire à l'égard de la nature primordiale, mais en tant que sol de l'expérience, il est « un domaine d'évidences originaires ${ }^{36}$ (ursprünglicher Evidenzen). Si la perception immédiate du monde de la vie n'est pas le donné le plus originaire, elle est première pour toute connaissance médiate relevant de cette sphère. Le monde de la vie rend possible tout sens scientifique puisque toute connaissance de lois porte sur le déroulement de phénomènes effectifs ou possibles. De ce point de vue, il est un sol originaire qui ne se donne pourtant pas dans son originarité voilée par le sens sédimenté. C'est donc comme si l'intuitivité première qui est celle du monde de la vie ne pouvait jamais se donner dans son originarité. C'est comme si la « pure expérience » elle-même dépassait déjà cette originarité. Ainsi s'opère l'inversion selon laquelle l'originaire ne peut être atteint que par une abstraction qui est celle de l'expérience. En ce sens, la nature originaire fonde toute expérience sans être elle-même un produit de l'expérience.

Dans certains textes des années 1920 et 1930, Husserl expose la notion phénoménologique d'induction comme procès qui appartient à la simple expérience. Lorsque la perception est décrite - comme ce sera le cas des Leçons du semestre d'été de 1927 (également intitulées Nature et esprit) ${ }^{37}$ en tant qu' « un procès d'une continuelle intention préalable (ou d'une continuelle anticipation) en remplissement continuel ${ }^{38}$, chaque perception est comprise comme "procès d'induction». Il est dit qu'une "situation antéprédicative » est déjà l'expérience de «ce qui est co-existant mais qui n'est pas soi-même perçu », c'est-à-dire une induction ${ }^{39}$. Dans les textes tardifs sur la problématique des «sciences de la nature », en réinterprétant le concept d'induction, Husserl décrit ce procès qui se trouve déjà en toute expérience immédiate. Et dans la Krisis, il écrit dans une note : « [...] Dans leurs anticipations de l'éprouvabilité, et du fait que toute perception directe elle-même inclut déjà des moments inductifs (anticipations des côtés de l'objet qui ne sont pas encore éprouvés), tout se trouve donc inclus dans le concept large d'“expérience" ou d'“induction" »". L'intuitivité du monde de la vie est donc celle de la pure expérience dans la mesure où elle inclut déjà des procès inductifs. Les substrats sensibles pré-donnés ne sont pas perçus en tant que des simples corps, mais la perception immédiate renvoie déjà à l'horizon non effectivement perçu : «De la façon la plus primitive, la

36. Ibid., p. 145 (traduction modifiée).

37. Texte dont la traduction française a été récemment publiée : Nature et esprit : leçons $d u$ semestre d'été 1927, trad. fr. Julien Farges, Paris, Librairie philosophique J. Vrin, 2017.

38. Cf. Ibid., p. 165 ; édition allemande: E. Husserl, Natur und Geist: Vorlesungen Sommersemester 1927, Boston-London, Kluwer, 2001, p. 140.

39. E. Husserl, Nature et esprit (1927), op. cit., p. 251 [256].

40. Krisis, p. 145 ; Hua VI, p. 130. 
certitude-de-l'être qui est celle de toute expérience simple est déjà une induction. Les choses "vues" sont toujours-déjà "plus" que ce que nous voyons d'elles "réellement et à proprement parler" " ${ }^{41}$. Par ailleurs, en tant qu'appartenant au monde pré-scientifique effectivement éprouvé, selon Husserl l' «induction quotidienne » précède l'induction scientifique. Une telle voie d'interprétation n'admet pas seulement une critique de l'objectivation de l'expérience, mais elle fournit les bases pour une nouvelle ontologie du monde la vie, fondée sur le mode de pré-donation de l'expérience intuitive qui est lui-même anticipable et a des moments inductifs.

D'après l'ouverture à l' ' expérience possible anticipée » que promeut l'induction, conçue comme méthode de vérification de l'expérience non immédiate, Husserl élabore une conception nouvelle de la nature. C'est ce que montre un manuscrit de la dernière période intitulé «Induction dans l'expérience du monde et constitution du monde orienté de l'expérience en tant que monde avec terre et ciel $»$, récemment traduit en langue française ${ }^{42}$. Comme le précisent les traducteurs, cet appendice fait partie d'un ensemble de textes datant de la dernière période de l'œuvre de Husserl dans lesquels il développe cette nouvelle conception ${ }^{43}$. L'intérêt de ce manuscrit est, selon eux, celui de fournir des précisions sur «la mise au jour de l'inductivité comme structure fondamentale de l'expérience ${ }^{44}$. Cette nouvelle conception de la nature qui se présente ne coïncide plus avec la couche de pure perception qui fonde toutes les autres, elle inclut également le sol de l'expérience aperceptive anticipatrice. Ainsi, dans un premier temps Husserl définit : «La nature en tant que noyau signifie ici la nature terrestre, en tant qu'elle s'étend continûment, selon une progression que l'on peut réitérer, dans les directions d'orientation [définies] depuis la place actuelle que l'on occupe sur terre ${ }^{45}$. Par ailleurs, par progression inductive nous pouvons en venir également au ciel et aux phénomènes célestes. Et peut-être aux parties inaccessibles de la terre? La portée d'une telle notion de nature est donc celle de la méthode inductive elle-même, ce sur quoi s'interroge Husserl : «Jusqu'où s'étend la vérifiabilité par l'expérience, jusqu'où s'étend l'induction et jusqu'où s'étend-elle en tant qu'elle est à vérifier? ${ }^{46}$.

41. Ibid., p. 59 [51].

42. E. Husserl, «Induction dans l'expérience du monde et constitution du monde orienté de l'expérience en tant que monde avec terre et ciel», Alter, 26, (2018), trad. fr. par Julien Farges et Laurent Perreau, p. 234-240.

43. Cf. J. Farges, L. Perreau, «Avant-propos des traducteurs», in «Induction dans l'expérience du monde et constitution du monde orienté de l'expérience en tant que monde avec terre et ciel », Alter, 26, (2018), trad. fr. par Julien Farges et Laurent Perreau, p. 234-240, p. 231.

44. Ibid., p. 232.

45. E. Husserl, «Induction dans l'expérience du monde et constitution du monde orienté de l'expérience en tant que monde avec terre et ciel », op. cit., p. 237.

46. Ibid., p. 236. 
La pensée du dernier Husserl ouvre ainsi à une nouvelle conception de nature qui n'est pas la couche qui fonde l'expérience, mais qui est le produit de l'expérience elle-même. En ce sens, elle ne se limite pas aux substrats sensibles pré-donnés dans la passivité, mais elle n'englobe pas non plus les significations pratiques et culturelles du monde de la vie. Dans la mesure où l'expérience ne se réduit pas au noyau de perception actuel, mais inclut l'horizon d'anticipation et de vérification, la portée de la nature peut être étendue à l'horizon indéterminé mais déterminable. La prise en compte de l'induction rapproche la nature de l'expérience concrète, sans l'élargir à la totalité de la réalité, ce qui ne la limite plus aux extrémités de son double sens. Il est ainsi révélé que la familiarité de l'expérience à laquelle renvoie le procès inductif pénètre la spatio-temporalité de la nature qui va donc au-delà de la pure perception: c'est la nature telle qu'elle se donne à l'expérience dans l'ouverture au déploiement de l'expérience possible par l'actualisation de potentialités intuitives. Dans la perspective d'un travail à venir, il se pose la question de savoir quel est le rôle de ce nouveau sens de la nature et plus particulièrement de l'induction dans la problématique de l'esthétique transcendantale.

\section{Références bibliographiques}

Begout, B., La Découverte du quotidien, Paris, Éditions Allia, 2005.

- L'Enfance du monde, Paris, Les Éditions de la Transparence, 2007.

- La Généalogie de la logique. Husserl, l'antéprédicatif et le catégorial, Paris, Vrin, 2000.

— « L'ontologie husserlienne du monde de la vie quotidienne, in J. Benoist \& B. Karsenti (dir.), Phénoménologie et sociologie, Paris, PUF, 2001, p. 83-104.

BENOIST, J., «Le monde pour tous: universalité et Lebenswelt chez le dernier Husserl », in Recherches husserliennes, vol. 5, 1996, p. 27-52.

BERMES, C., " "Monde" et "monde vécu" dans la philosophie au XIX siècle et dans la philosophie husserlienne », Études phénoménologiques, 37-38, (2003), p. 175-195.

Bernet, R., Kern, I., MARBACH, E., Husserl, Darstellung seines Denkens, Hamburg, F. Meiner, 1989, chapitre 9: «Die Lebenswelt als Grundlagenproblem der objektiven Wissenschaften und als universales Wahrheits - und Seinproblem », trad. fr. par Philippe Cabestah, Alter, 6, (1998), p. 419-431.

Bernhard, R., Husserls Phänomenologie der materiellen Natur, Frankfurt am Main, V. Klostermann, 1990.

BRISART, R., «L'expérience perceptive et son passif. À propos des sensations dans le constructivisme de Husserl», Philosophie, 119, (2013/4), p. 33-63. 
FARGES, J., « Monde de la vie et primordialité chez Husserl. Les ambiguïtés de la conception phénoménologique de l'esthétique transcendantale », Philosophie, 108, (2011/1), p. 15-34.

HUSSERL, E., Erfahrung und Urteil: Untersuchungen zur Genealogie der Logik, Hrsg. von L. Landgrebe, Claassen \& Goverts, Hamburg, 1948 ; Expérience et jugement, trad. fr. par Denise Souche-Dagues, Paris, Presses Universitaires de France, 2001.

- Husserliana IV. Edmund Husserl gesammelte Werke, Ideen zu einer reinen Phänomenologie und phänomenologischen Philosophie. 2, Phänomenologische Untersuchungen zur Konstitution, Haag, Martinus Nijhoff, 1952; Recherches phénoménologiques pour la constitution, trad. fr. Éliane Escoubas, Paris, Presses Universitaires de France, 2004.

- Husserliana VI. Edmund Husserl gesammelte Werke, Die Krisis der Europäischen Wissenschaften und die Transzendentale Phänomenologie, Haag, Martinus Nijhoff, 1962 ; La Crise des sciences européennes et la phénoménologie transcendantale, trad. fr. G. Granel, Gallimard, 1976.

- «Induktion in der Welterfahrung und die Konstitution der orientierten Erfahrungswelt als Welt mit Erde und Himmel », in Die Lebenswelt. Auslegungen der vorgegebenen Welt und ihrer Konstitution. Texte aus dem Nachlass (1916-1937), R. Sowa (éd.), Dordrecht, Springer, 2008 ; Husserliana XXXIX, Beilage XIII, p. 184-189 ; «Induction dans l'expérience du monde et constitution du monde orienté de l'expérience en tant que monde avec terre et ciel », Alter, 26, (2018), trad. fr. par Julien Farges et Laurent Perreau, p. 234-240.

- Natur und Geist : Vorlesungen Sommersemester 1919, Boston-London, Kluwer, 2002.

- Natur und Geist: Vorlesungen Sommersemester 1927, Boston-London, Kluwer, 2001 ; Nature et esprit : lecons du semestre d'été 1927, trad. fr. Julien Farges, Paris, Librairie philosophique J. Vrin, 2017.

JANSSEN, P., Geschichte un Leenswelt, ein Beitrag zur Diskussion von Husserls Spätwerk, Nijhoff, Den Haag, 1970.

KERN, I., «Die Lebenswelt als Grundlagenproblem der objektiven Wisenschaften und als universales Wahrheits und Seinsproblem », in R. Bernet, I. Kern, E. Marbach (Hrsg.), Edmund Husserl, Darstellung seines Denkens, Hambourg, F. Meiner, 1989, p. 199-208 ; trad. fr. par $\mathrm{Ph}$. Cabestan, in Alter. Revue de phénoménologie, 6, 1998, p. 419-431.

PRADELlE, D., L'Archéologie du monde. Constitution de l'espace, idéalisme et intuitionnisme chez Husserl, Dordrecht/Boston/London, Kluwer, 2000. 